
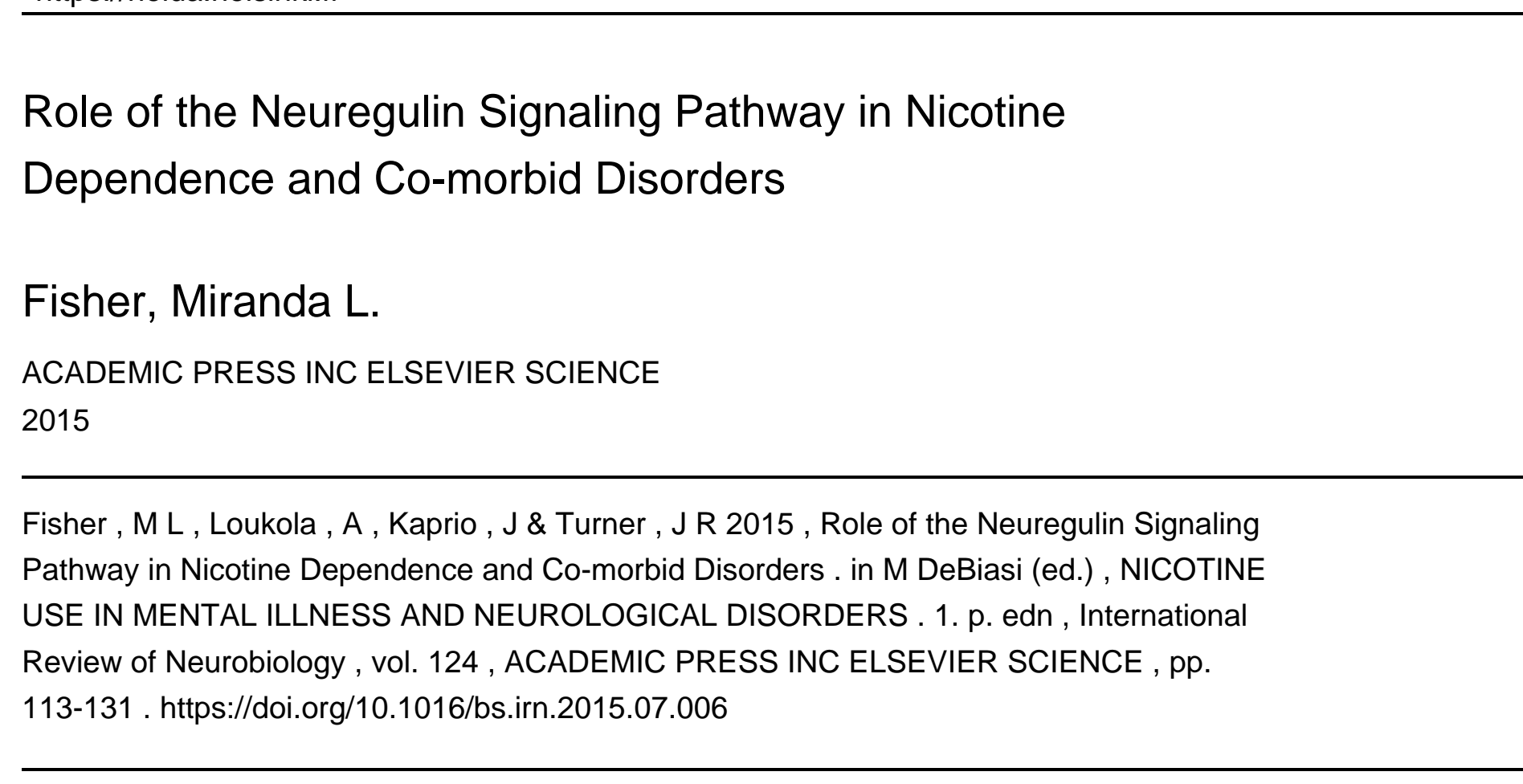


\section{Role of the Neuregulin Signaling Pathway in Nicotine Dependence and Co-morbid Disorders}

Miranda L. Fisher*, Anu Loukola ${ }^{\dagger}$, J aakko Kaprio ${ }^{\dagger,\{,\}}$, Jill R. Turner*,I *D epartment of D rug D iscovery and Biomedical Sciences, South C arolina C ollege of Pharmacy, U niversity of South Carolina, Columbia, South Carolina, U SA

${ }^{\dagger} \mathrm{D}$ epartment of Public H ealth, Faculty of M edicine, U niversity of $\mathrm{H}$ elsinki, H elsinki, Finland

${ }_{\mathrm{N}}^{\mathrm{N}}$ ational Institute for $\mathrm{H}$ ealth and $\mathrm{W}$ elfare, $\mathrm{H}$ elsinki, Finland

Institute for M olecular M edicine Finland FIM M , U niversity of H elsinki, H elsinki, Finland

${ }^{1}$ C orresponding author: e-mail address: jiturner@ sccp.sc.edu

\section{Contents}

1. Introduction

2. Known Mechanisms of Neuregulin-ErbB Signaling 117

$\begin{array}{ll}2.1 \text { Overview } & 117\end{array}$

2.2 Neuregulin Binding and ErbB Dimerization 118

2.3 Effects of Alternative Splicing of the ErbB4 Receptor 119

2.4 Neuregulin-ErbB4 Effects on NMDA Receptors 120

2.5 Acetylcholine Receptor Inducing Activity: Modulation of nAChR Expression 120

3. NRG3: Relevance in Smoking Behavior and Co-morbid Disorders

3.1 Potential Role of NRG3 in Nicotine Withdrawal and Smoking Cessation

Outcome

3.2 NRG3 in Schizophrenia

3.3 NRG3: Possible Mechanisms Underlying Co-morbidity 122

4. ERBB4: Relevance in Smoking Behavior and Co-morbid Disorders 123

4.1 Association Between ERBB4 and Nicotine Dependence 123

4.2 ErbB4-NMDA Receptor Interactions in Schizophrenia and Possible

Relevance for Co-morbidity with Nicotine Dependence 124

5. Summary

References

\section{Abstract}

Smoking is currently the leading cause of preventable death in the United States and is responsible for over four million deaths annually worldwide. Therefore, there is a vast clinical unmet need with regards to therapeutics targeting smoking cessation. This is even more apparent when examining smokers co-morbid with psychiatric illness, as rates of smoking in this population are 4 higher than in the general population. 
Examining common genetic and molecular signaling pathways impinging upon both smoking behavior and psychiatric illness will lead to a better understanding of co-morbid disorders and potential development of novel therapeutics. Studies have implicated the Neuregulin Signaling Pathway in the pathophysiology of a number of psychiatric illnesses. Additionally, recent studies have also shown an association between the Neuregulin Signaling Pathway and smoking behaviors. This review outlines basic mechanisms of the Neuregulin Signaling Pathway and how it may be exploited for precision medicine approaches in treating nicotine dependence and mental illness.

\section{INTRODUCTION}

T obacco smoking is still the leading cause of preventable death in the U nited States even years after the discovery of multiple smoking cessation therapies. The main addictive component in cigarette smoke is nicotine (U nited States. D epartment of the Army. O ffice of the Surgeon General, 1988), which drives the reinforcement behind smoking behavior. W ith global smoking-related mortality reaching nearly six million deaths annually ("W H O urges more countries to require large, graphic health warnings on tobacco packaging: the W HO report on the global tobacco epidemic, 2011 examines antitobacco mass-media campaigns," W H O , 2011), there is a high demand for targeted therapeutics that successfully aid smokers to quit. Several smoking cessation pharmacotherapies are available, including nicotine replacement therapy, prescription medication such as bupropion (originally designed as an antidepressant) and the nicotinic acetylcholine receptor (nAChR) partial agonist varenicline (Cummings \& M ahoney, 2006; Jorenby et al., 2006); how ever, the success rate of such therapies after 1 year is at best only $20-25 \%$ (G onzales et al., 2006). In comparison, approximately $3 \%$ of individuals trying to quit without any pharmacotherapies are still abstinent after 6 months (H ughes et al., 1992). The majority of smokers would like to quit and are aware of the risks of smoking, but are unable to do so. The positive reinforcing effect of nicotine is an important determinant of cessation failure; however, it is not the only factor that should be taken into account. The significant aversive withdrawal symptoms that occur during abstinence are also considered a major determinant of high relapse rates (Le Foll \& Goldberg, 2009).

W ithdrawal symptomsare relatively well-characterized and include both cognitive and affective symptoms. These symptoms primarily include 
depressed mood states, anxiety, irritability, concentration difficulties, and craving (Hughes, 2007). It is suggested that withdrawal symptom severity is a more valid indicator of smoking cessation outcome than nicotine intake or dependence (W est, $\mathrm{H}$ ajek, \& Belcher, 1989). Of these aversive withdrawal symptoms, a common affective symptom is depression. Interestingly, depressed mood is also associated with nicotine dependence, but it is not known whether depression predisposes an individual to begin smoking or whether depression develops during the course of nicotine dependence.

B roadly, nicotine dependence is highly co-morbid with several psychiatric illnesses and other substance use disorders, which further complicates smoking cessation. However, the relationship between nicotine use and mental disorders is still elusive and debatable (M oylan, Jacka, Pasco, \& Berk, 2012). It has been suggested that nicotine is used in an effort to self-medicate symptoms occurring in psychiatric illnesses such as schizophrenia (R oyal College of Physicians of London \& R oyal College of Psychiatrists, 2013), i.e., smoking would primarily be a consequence of the psychiatric disease. The second possible explanation for the co-morbidity is that smoking is itself a cause of psychiatric illness; the evidence for this is variable and depends on conditions being examined. For example, growing evidence supports the causal role of smoking in the etiology of depression (B reslau, Peterson, Schultz, Chilcoat, \& Andreski, 1998; Kendler et al., 1993; Pasco et al., 2008). However, evidence must come from wellconducted prospective epidemiological studies, within-family studies, or $M$ endelian randomization studies using genetic markers to test causality, as randomized clinical trials cannot be used to test this hypothesis. The third potential reason for the co-morbidity may be that there are underlying genetic factors in common to specific mental disorders and smoking-related phenotypes, including nicotine dependence and withdrawal. A prime candidate for this third explanation is the co-morbidity observed between nicotine dependence and schizophrenia.

Within the co-morbid population, overall prevalence of smoking in schizophrenia patients is higher than in patients with other psychiatric conditions (Dickerson et al., 2013). Strikingly, high smoking prevalences, $60-90 \%$, have been reported in schizophrenia patients (Dickerson et al., 2013; M atthews, W ilson, \& M itchell, 2011; Zabala et al., 2009), compared to the approximately $18 \%$ prevalence rate in the general U.S. population ( Jamal et al., 2014). In addition to being more frequently current smokers, schizophrenia patients typically smoke more, are more likely nicotine dependent, and are less likely to succeed in quitting (Addington \& 
el-Guebaly, 1998; George et al., 2002; Ziedonis \& George, 1997). H owever, the association of smoking with schizophrenia is not universal. For example, among $C$ hinese women with schizophrenia, the prevalence of smoking was only sightly higher than in the general Chinese population (H ou et al., 2011; X u et al., 2014). H owever, this finding could reasonably be due to a greater percentage of smokers in the $C$ hinese population (28.1\%) (Li, H sia, \& Y ang, 2011) as compared to the $20 \%$ of A mericans. N onetheless, the strength and consistency of the association over the W estern world suggests that there may also be an underlying biological basis for it. Furthermore, given the differences in genetic architecture between major human ancestry groups, the findings in $\mathrm{C}$ hinese patients do not exclude a genetic contribution in European ancestry populations.

The neurodevelopmental theory of schizophrenia suggests that genetic and/or environmental factors negatively affect brain development during critical neural development milestones (R apoport, Addington, Frangou, \& Psych, 2005). These in turn are responsible for the biochemical alterations observed in people diagnosed with the disease ( $M$ arenco, W einberger, \& Schreurs, 2003). B reaking down the symptom profile of schizophrenia into several disease- relevant endophenotypes hasenabled investigation of the role of specific risk genesthat impact behavioral and biological components of this disease phenotype (Braff, Freedman, Schork, \& Gottesman, 2007; $W$ addington et al., 2007; W alters \& $O$ wen, 2007). For example, linkage and association studies have resulted in several candidate genes such as D T N BP 1, D ISC 1, N R G 1, and N R G 3 . O ne of the most promising susceptibility genesfor schizophreniaisN R $G 3$ due to the observation that structural and polymorphic variations of thisgene are associated with aw ide spectrum of neurodevelopmental disorderswith phenotypesencompassing developmental delay, impairment of cognition, and autism (Balciuniene et al., 2007). T his genetic variation is due to recurrent microdeletions of chromosome 10q22q23 that involve the N R G 3 gene and al so shows linkage to schizophrenia in A shkenazi Jewish and $\mathrm{H}$ an $\mathrm{C}$ hinese populations (Fallin et al., 2003; Faraone et al., 2006). A noncoding genetic variation in N R G 3 hasalso been observed asaputativerisk factor for schizophrenia(C hen etal., 2009; M orar etal., 2011; Sonuga-Barke et al., 2008; W ang et al., 2008). A dditionally, genetic association studies show multiple genes and epistatic locus interactions (Benzel et al., 2007) within the N R G-ErbB signaling pathw ay that increases the risk for schizophrenia. These multiplegenesencodefor N R G 3, N R G 1, ER BB 4, and $A K T 1$, suggesting thissignaling cascade may represent a pathogenic network occurring in schizophrenia. 














